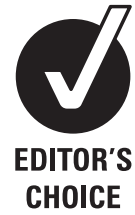

CHOICE

\begin{abstract}
${ }^{1}$ Division of Neurosurgery, State University of New York at Stony Brook, Stony Brook, New York, USA ${ }^{2}$ Division of Neurosurgery, ENERI Medical Institute, Equipo de Neurocirugía Endovascular y Radiología Intervencionista, Buenos Aires, Argentina ${ }^{3}$ Division of Neurosurgery and Neuroradiology, National Institute of Neurosurgery, Budapest, Hungary ${ }^{4}$ Division of Neurosurgery, Royal University Hospital, Saskatchewan, Canada ${ }^{5}$ Division of Neurosurgery, Barrow Neurological Institute, Phoenix Arizona, USA ${ }^{6}$ Division of Neuroradiology and Neurosurgery, New York University, New York, New York, USA
\end{abstract}

Correspondence to: Dr D Fiorella, Department of Neurosurgery, Cerebrovascular Center, State University of New York at Stony Brook, Health Sciences Center T-12 080, Stony Brook, New York 11794-8121, USA; dfiorell@ gmail.com

Received 1 April 2009 Accepted 3 April 2009

\title{
Curative cerebrovascular reconstruction with the Pipeline embolization device: the emergence of definitive endovascular therapy for intracranial aneurysms
}

\author{
D Fiorella, ${ }^{1}$ P Lylyk, ${ }^{2}$ I Szikora, ${ }^{3}$ M E Kelly, ${ }^{4}$ F C Albuquerque, ${ }^{5}$ C G McDougall, ${ }^{5}$ \\ P K Nelson 6
}

\section{ABSTRACT:}

Endovascular, endosaccular, coil embolization has emerged as an established therapy for both ruptured and unruptured cerebral aneurysms. However, many aneurysms are not cured using conventional endovascular techniques. Coil embolization often results in incomplete aneurysm occlusion or recanalization in the ensuing months after treatment. The Pipeline embolization device (PED; Chestnut Medical) represents a new generation endoluminal implant which is designed to treat aneurysms by reconstructing the diseased parent artery. Immediately after implantation, the PED functions to divert flow from the aneurysm, creating an environment conducive to thrombosis. With time, the PED is incorporated into the vessel wall as neointimal-endothelial overgrowth occurs along the construct. Ultimately, this process results in the durable complete exclusion of the aneurysm from the cerebrovasculature and a definitive endoluminal reconstruction of the diseased parent artery.

\section{CONTEMPORARY ENDOVASCULAR THERAPY FOR INTRACRANIAL ANEURYSMS}

The International Subarachnoid Aneurysm Trial, ${ }^{1} 2$ the Barrow Ruptured Aneurysm Trial $^{3}$ and the California unruptured aneurysm database ${ }^{4}$ have provided evidence that endovascular therapy has equipoise with, and in appropriately selected patients may be superior to, open neurosurgical clipping. These studies have supported the approach of a prospective "endovascular right of first refusal" with respect to the treatment of intracranial aneurysms.

However, contemporary endovascular therapies, even when incorporating state of the art adjunctive devices (self-expanding intracranial microstents, hypercompliant temporary occlusion balloons and deflectable tipped microcatheters), are frequently challenged by an unfavorable anatomical configuration and neck geometry of the aneurysm. ${ }^{5-7}$ Although treatment of these lesions can frequently be accomplished, the final result is often suboptimal, sometimes yielding only partial aneurysm occlusion. Even when an "acceptable" level of aneurysm occlusion is achieved initially, the result may not be durable with lesion recanalization occurring over the ensuing months.

Raymond and colleagues ${ }^{8}$ critically reviewed their single center experience with 501 aneurysms treated with coil embolization. They found that the majority of the aneurysms treated were not angiographically cured by coil embolization. They reported a complete angiographic occlusion rate of only $38.3 \%$ at 1 year follow-up. Also, once treated, the angiographic result achieved with coiling was not durable in up to one-third of the cases, with $33.6 \%$ of treated aneurysms showing recanalization over the course of 1 year. One-fifth of aneurysms treated $(20.7 \%)$ had recurrences (termed "major recurrences") which were of a magnitude that could accommodate the placement of one or more additional coils. Once retreated, aneurysms also demonstrated a propensity to recur a second time, with $48.6 \%$ requiring one or more additional retreatments after the subsequent re-embolization.

These failures of traditional endosaccular aneurysm therapy are amplified with aneurysms of larger sizes and those that incorporate longer segments of the diseased parent artery. When looking at large aneurysms $\left(>10 \mathrm{~mm}\right.$ ), Raymond and colleagues ${ }^{8}$ reported recurrence rates of approximately $50 \%$. Other authors have observed a similar trend towards high rates of recurrence for larger wide necked aneurysms. ${ }^{9}$ In a recent review on this topic, van Rooij and Sluzewski ${ }^{10}$ recommend parent artery occlusion as the treatment of choice for giant aneurysms. They suggest that when deconstruction is not possible and a constructive endosaccular treatment strategy is undertaken, that this approach be essentially considered a "staged treatment" in which the neurointerventionist prospectively plans to coil and recoil the aneurysm each time it occurs.

Although some investigators, including ourselves, have advocated the application of the currently commercially available self-expanding intracranial microstents to augment the durability of coil embolization for these wide necked large and giant aneurysms, there are no reliable data to support this practice. ${ }^{6} 1112$

\section{WHY DOES COILING FAIL?}

There are a number of potential reasons that endosaccular aneurysm occlusion fails to achieve a curative durable treatment in the majority of cases. Firstly, it is difficult, if not impossible, in many cases to anatomically reconstruct a large segmental parent artery defect with randomly distributed strands of platinum coil. Almost invariably, even with the most meticulous technique, there are 

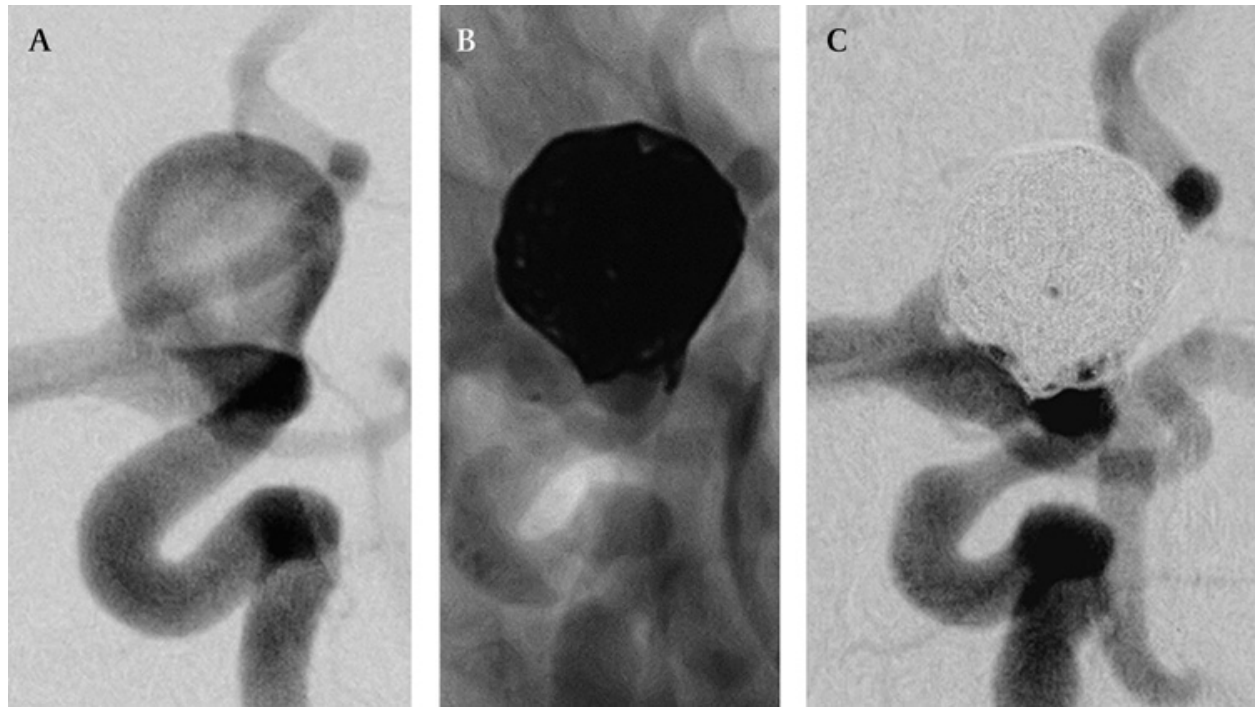

Figure 1 Large wide necked right internal carotid artery, carotid-ophthalmic segment aneurysm (A). Native (B) and subtracted (C) images after coil embolization with a balloon assisted technique. Despite aggressive packing of the aneurysm, there is still an irregular interface between the coil mass and the parent artery, with several gaps in the coil mass near the aneurysm neck. In addition, the coil mass within the fundus of the aneurysm has several gaps, some of which still fill with contrast at the conclusion of the procedure. This case demonstrates the difficulty in achieving a homogeneous reconstruction of the entire aneurysm neck with randomly positioned coils. It is also evident that much of the aneurysm fundus is not filled with embolic material, reflecting the $20-40 \%$ packing density typically achieved with platinum coils.

numerous small gaps at the interface between the coil loops and the parent artery which allow blood flow through the interstices of the coil mass and into the aneurysm neck (fig 1). Secondly, even using the newest generation platinum coils in concert with commercially available adjunctive devices, the best achievable packing densities typically range between $20 \%$ and $40 \%{ }^{13}{ }^{14}$ Thus even after aggressive coil embolization, the majority of the aneurysm volume is filled with soft thrombus rather than more structurally secure platinum coils (fig 1). Thirdly, the diseased parent artery segment giving rise to the aneurysm is not necessarily addressed by filling the saccular component with coils. This can result in a regional recurrence of the aneurysm arising from an adjacent portion of the circumferentially or segmentally diseased parent artery (fig 2).

\section{WHAT IS THE PIPELINE EMBOLIZATION DEVICE?}

The PED is a self-expanding, cylindrically shaped, endovascular construct composed of 48 braided strands of cobalt chromium and platinum (fig 3 ). The individual strands measure between 28 and $33 \mathrm{~mm}(0.0011-0.0013$ inches $)$ in outer diameter. A single PED has 30-35\% metal surface area coverage when fully deployed within an appropriately size matched vessel. ${ }^{15} 16$ Multiple devices can be deployed within each other (telescoped) to create a composite endovascular construct. This telescoping technique can be used either to increase the overall length of the construct or to augment the degree of metal surface area coverage over a particular segment. ${ }^{16-19}$ The degree of metal surface area coverage can also be manipulated by the technique of device deployment and the sizing of the device relative to the parent artery.
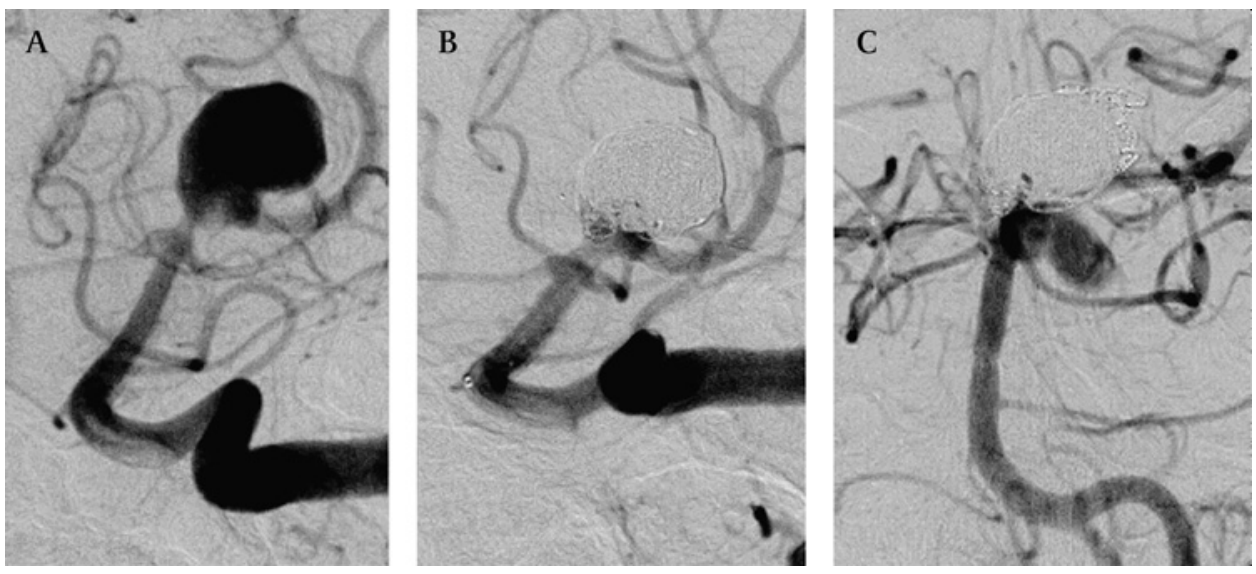

Figure 2 Large basilar apex aneurysm (A), treated with stent supported coil embolization. Post-treatment subtracted image (B) immediately after treatment demonstrates near complete occlusion of the aneurysm with minimal residual filling in the region of the aneurysm neck. At follow-up, the coiled portion of the aneurysm remains occluded and the coil mass is not distorted or compacted. However, an adjacent segment of the diseased vessel wall has blown out into a large aneurysm. This case demonstrates the failure of coil embolization and the conventional self-expanding stents to address the issue of segmental parent artery disease. 


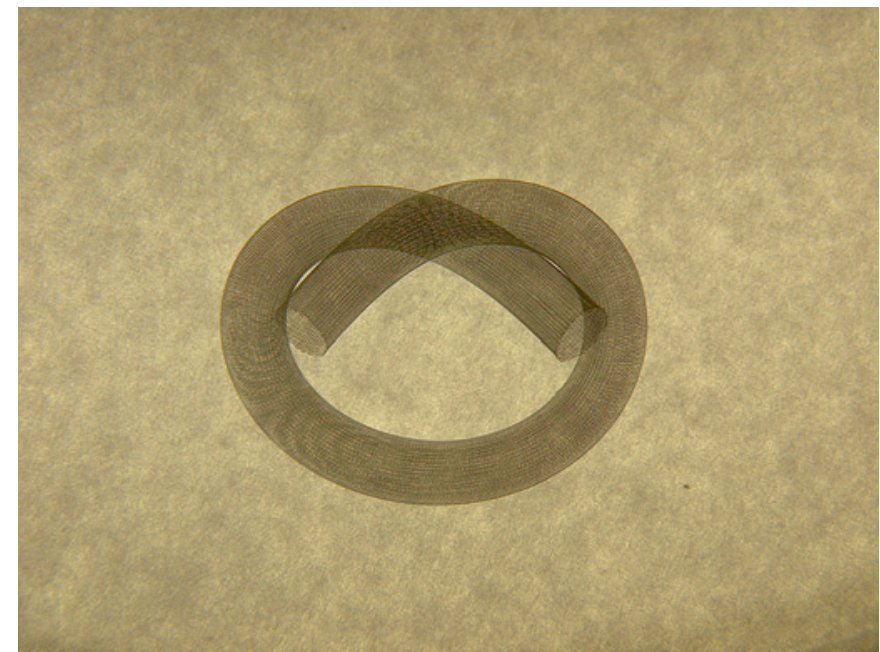

Figure 3 High resolution photograph of a Pipeline embolization device (PED). The PED represents a conglomerate structure composed of 48 individual braided strands of platinum and cobalt chromium. When fully deployed, the construct remains very flexible and can be tied into a "pretzel" configuration without kinking or ovalizing. When constrained within the microcatheter over the delivery wire, the construct elongates approximately 2.5 times its fully deployed length.

The PED is mounted on a delivery microwire and constrained within a removable sheath. The PED is loaded into and delivered through a standard 0.027 ID microcatheter (Hi-Flo Renegade (Boston Scientific, Fremont, California, USA), Mass Transit (Cordis Neurovascular/Johnson and Johnson, Warren, New Jersey, USA) or Marksman (Chestnut Medical Inc, Menlo Park, California, USA)). The available sizes range from 2.5 to $5.0 \mathrm{~mm}$ in diameter and) $10-20 \mathrm{~mm}$ in length. Any anatomical location which can be accessed with a 0.027 inch ID microcatheter can be reconstructed with the PED. The device is extremely flexible when deployed and conforms to the native anatomy with very little anatomical distortion, regardless of the regional tortuosity.

Incorporation of platinum strands within the body of the construct imparts a functional degree of radio-opacity to the device which is typically visible throughout its length when implanted in situ. This radio-opacity represents a significant working advantage in comparison with the existing radiolucent self-expanding intracranial stents, particularly with building multi-device constructs. At the same time, the degree of radioopacity does not obscure the regional soft tissues on CT imaging. In fact, CT angiography (CTA) is an effective means by which to follow aneurysms after treatment with the PED (fig 4).

\section{THEORETICAL BASIS OF PARENT ARTERY RECONSTRUCTION WITH PIPELINE}

Parent artery reconstruction with Pipeline is conceptually much closer to surgical aneurysm clipping than is endosaccular coil occlusion. Fundamentally, both techniques function to exclude the abnormal aneurysmal segment of the parent artery by bridging normal artery with normal artery. The PED achieves this by providing an endovascular scaffolding on which a structurally secure de novo artery can be circumferentially reconstructed across the diseased segment. Ultimately, the neointima and endothelium which cover the construct become contiguous with the endoluminal surfaces of the normal distal and proximal parent artery, thereby excluding the abnormal artery and bridging from normal vessel to normal vessel (fig 5).

Reconstruction starts immediately after the construct is in place and then evolves over a period of weeks to months. The process is characterized by a gradual transition from flow disruption to complete angiographic occlusion and ultimately to curative anatomical restoration. The stages of this transition can be characterized as follows:

1. Mechanical-anatomical: flow disruption (immediate). After the construct is in place, the physical barrier along the neck of the aneurysm results in the disruption of both inflow and outflow with redirection of the primary vector of blood flow along the course of the anatomically reformed parent artery. This effect would be expected to considerably reduce the velocity of the inflow jet as well as the level of shear stress on the aneurysm wall (fig 6). At this point, while the aneurysm may still fill with contrast, many of the
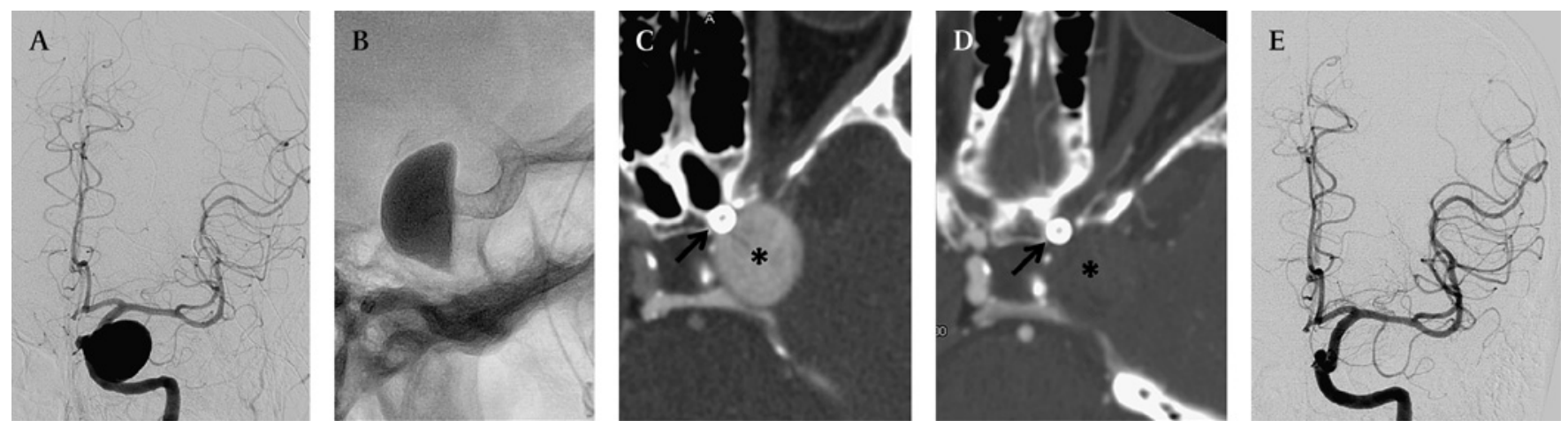

Figure 4 A 63-year-old female with a giant left internal carotid artery, cavernous segment aneurysm undergoing treatment with PED. Angiography from a catheter positioned within the left internal carotid artery (A) shows the giant cavernous aneurysm. A lateral native image $(B)$ after the construct has been placed demonstrates contrast stasis within the dependant aspect of the aneurysm. The construct is easily visualized on fluoroscopy through the osseous structures of the skull base. Computed tomographic angiogram (CTA) obtained on post-operative day (POD) 1 shows the construct in place within the carotid artery and persistent filling of the aneurysm with contrast (C). There is no significant artifact created by the construct. The surrounding anatomy is easily evaluated. On POD 11, the patient experienced worsening of her headaches and left retro-orbital pain along with diplopia, A POD 12 CTA shows complete thrombosis of the aneurysm (D). The interval exacerbation of symptoms was attributed to transient inflammation and mass effect attributable to the process of aneurysm thrombosis. These symptoms completely resolved over the next 2 months. Follow-up left carotid angiography (E) performed 5 months after treatment shows complete occlusion of the aneurysm with anatomical remodeling of the carotid artery. 

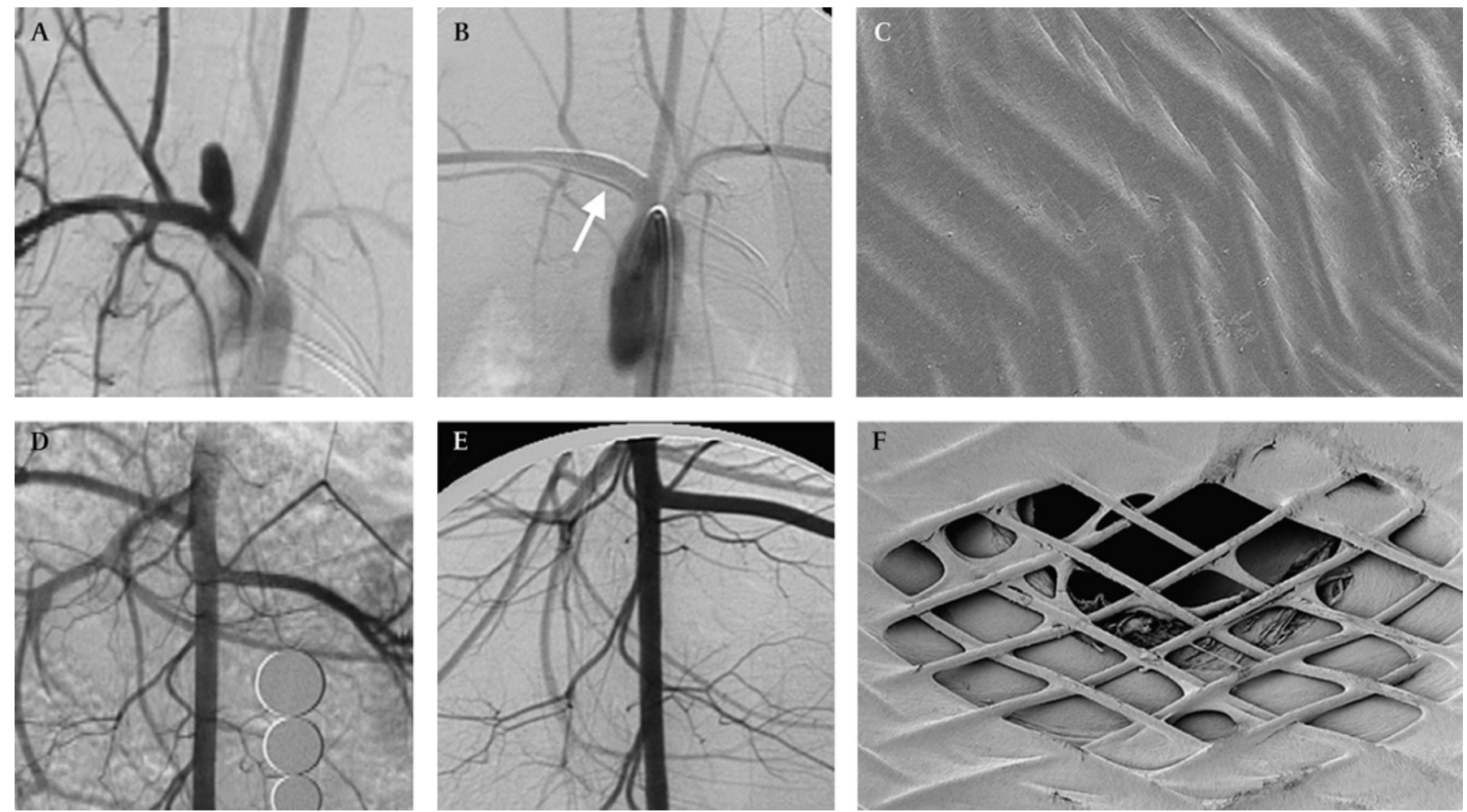

Figure 5 Images from an experiment performed by Kallmes and colleagues ${ }^{16}$ (with permission) using the Pipeline embolization device (PED) to treat experimental aneurysms in the rabbit. Angiography from an innominate projection shows an experimental aneurysm arising from the proximal innominate (A). Immediately following placement of a PED device, the aneurysm shows markedly reduced contrast opacification (B). At 6 month followup angiography $(C)$, the aneurysm has progressed to complete angiographic occlusion while the adjacent vertebral artery (also covered by the PED) remains patent. A PA image of the abdominal aorta performed 6 months after PED implantation within this vessel shows that all of the lumbar artery side branches covered by the PED remain patent (figure and legend are reproduced with permission from Endovascular Today).

factors which would be expected to contribute to local mass effect, progressive growth and rupture are very likely reduced, if not altogether eliminated (fig 6). In some cases, the presenting symptoms of mass effect or headache can improve considerably immediately after the procedure.

2. Physiological: aneurysm thrombosis (days to weeks). Following placement of the construct and disruption of intra-aneurysmal flow, the aneurysm typically begins to thrombose and angiographic imaging (conventional or cross sectional) demonstrates a progression towards complete occlusion (fig 6). Cross sectional imaging demonstrates a thrombus mass within the aneurysm. The rate of this thrombosis seems to vary significantly with aneurysm size, location and degree of PED overlap within the construct. Clinically, the presenting symptoms may progress at this point due to local mass effect or transmural inflammatory changes secondary to the thrombosis. In some cases, new symptoms or an exacerbation of pre-existing symptoms (eg, headache or cranial neuropathy) may manifest during this time (fig 4).

3. Biological: construct endothelialization and thrombus resorption (months). After flow into the aneurysm is eliminated, the construct becomes endothelialized, forming a permanent biological seal across the segmentally diseased parent artery. This process represents the culmination of the reconstruction from an endovascular standpoint with neoendothelium growing along the construct to bridge the normal proximal and distal parent artery segments. After the aneurysm is completely excluded from the circulation, the thrombus mass then begins to resorb. Eventually the entire aneurysm mass begins to collapse around the periphery of the construct, representing an evolution towards the restoration of both normal anatomy and physiology (fig 7). Those symptoms related to aneurysm mass effect (which remain reversible) typically resolve at this point.

The paradoxical counterpart to the efficacy of Pipeline in closing aneurysms is the relative safety of construct deployment over regional eloquent perforators and branch vessels which remain patent. As discussed above, this phenomenon represents the interaction of the Pipeline with the underlying anatomy, physiology and vascular biology. Aneurysm filling is solely dependent on the trajectory of blood flow at the aneurysm-parent vessel interface. The anatomy of the parent artery-aneurysm complex, defines the inflow and outflow jets, the intra-aneruysmal flow velocity and the shear forces on the aneurysm wall. Once the trajectory of blood flow is redirected within the parent artery and disrupted within the aneurysm, aneurysm filling slows to the point at which a prothrombotic environment is created. Having established this level of flow disruption, the aneurysm typically progressively thromboses over the ensuing days to weeks.

Flow into the regional perforator and branch arteries is governed by factors which are different than those which drive flow into an aneurysm. Flow into branch vessels is driven by a pressure gradient between the high pressure parent artery and the lower pressure branches which perfuse end organs and 

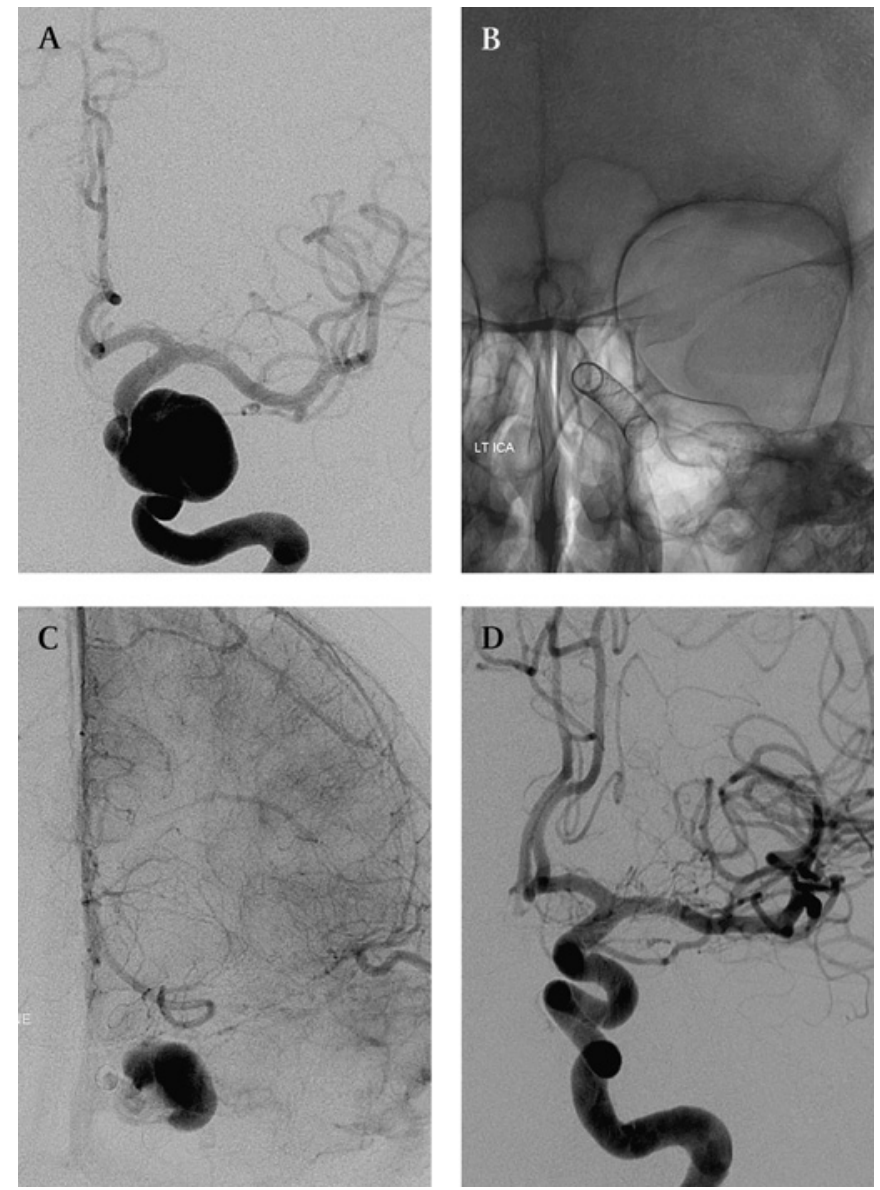

Figure 6 A 51-year-old female with acute onset diplopia and left ptosis. Intitial PA angiogram (A) shows a large symptomatic cavernous segment aneurysm involving the left internal carotid artery (ICA). She had failed a balloon test occlusion of the left ICA, and for this reason, she underwent treatment with PED. A native image (B) shows the in situ PED construct (three devices - $3.75 \mathrm{~mm} \times 20 \mathrm{~mm} ; 4.0 \mathrm{~mm} \times 16 \mathrm{~mm} ; 4.25 \mathrm{~mm} \times 14$ $\mathrm{mm}$ ) within the parent artery, across the aneurismal segment. Immediately after treatment, a subtracted venous phase image (C) shows stasis of constrast within the aneurysm, now persisting late into the venous phase. Follow-up subtracted PA (D) angiogram obtained at 6 months shows curative anatomical reconstruction of the parent artery with complete occlusion of the aneurysm.

ultimately drain into the very low pressure venous system. As such, these vessels can conceptually be envisioned as small siphons which draw blood flow from the parent artery. Under these circumstances, in which a physiological artery to venous pressure gradient exists, more than $50 \%$ occlusion of the luminal surface area is required before flow starts to diminish significantly. $^{20} 21$ The maintenance of this pressure gradient forms the foundation for blood flow, vascular patency and ultimately device endothelialization. Histological evaluation of Pipeline 6 months after implantation into the rabbit aorta validates this concept, demonstrating a homogeneous sheet of neoendothelium and neointima growing across the reconstructed vessel lumen. This continuous smooth sheet of tissue is interrupted only by rounded funnel-like defects which lead to the orifices of the regional branches (fig 5). Thus flow not only maintains patency in the short term but it also preserves patency in the long term as it governs this process of construct endothelialization.
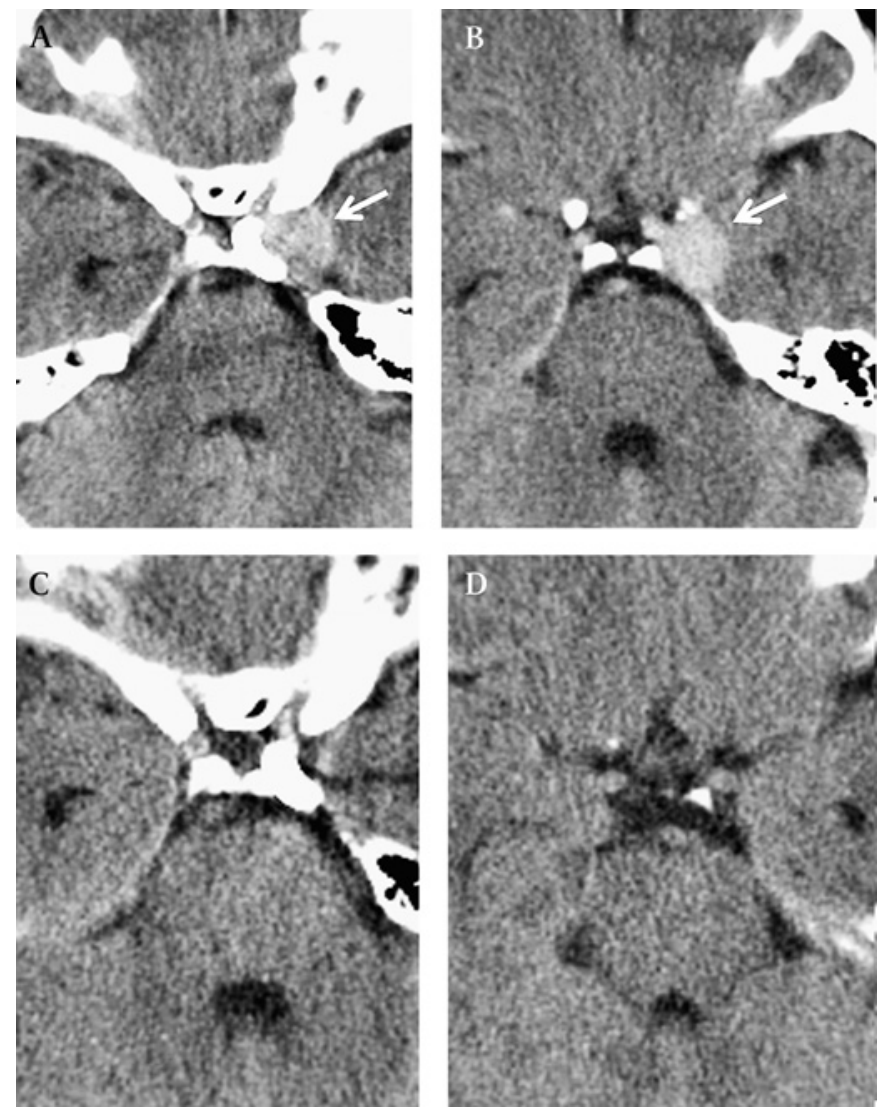

Figure 7 A 51-year-old female with acute onset diplopia and left ptosis, treated with a Pipeline embolization device (PED) (see fig 6). CT angiography images $(A, B)$ from postoperative day 1 demonstrate the PED construct in place within the parent internal carotid artery. One day after the procedure the aneurysm still fills with contrast and exerts a mass effect on the medial aspect of the left temporal lobe (arrows). CT images from the 6-month follow-up (C, D) demonstrate resolution of the aneurysm which has collapsed around the outside of the construct as the intra-aneruysmal thrombus has been resorbed. A mass effect on the medial temporal lobe has resolved. The CSF spaces around the left aspect of the suprasellar cistern are now reconstituted. At the time of the 6-month follow-up CT, her ocular symptoms had completely resolved.

This pressure gradient may not be as great in vessels that have robust competitive collateral flow. This is most frequently seen when constructs are built over the ophthalmic artery which occasionally occludes when covered by multiple devices. In these situations, competitive collateral flow from the external carotid artery may create a "flow equalization point" resulting in slow flow or proximal occlusion of the ophthalmic artery which will then fill distally from the external circulation. As PED is applied with increasing frequency across intracranial branch vessels which communicate via the circle of Willis, it is likely that similar physiological and hemodynamic patterns of reorganization will be observed.

Thus the porosity of a judiciously built PED construct strikes a delicate balance by providing enough flow diversion to initiate the cascade towards curative aneurysm occlusion yet permitting enough flow across the construct such that the regional eloquent branch vessels (which lack sufficient collateral flow) may remain patent. This balance is essential for any flow diverting device to be applied safely and effectively within the cerebrovasculature. 


\section{EXPERIENCE WITH THE PIPELINE EMBOLIZATION DEVICE}

The PED has been used to treat aneurysms in over 200 humans to date and the number continues to increase weekly. Experience with the device so far can be categorized as follows:

1. Individual North American compassionate use cases (January 2007-present)

2. The Pipeline Embolization Device in the Intracranial Treatment of Aneurysms (PITA) Trial (January 2007-November 2007)

3. The Buenos Aires Pipeline experience (March 2006-present)

4. The US Pipeline trials (October 2008-present)

a. Complete Occlusion of COilable intracranial Aneurysms (COCOA) trial

b. Pipeline for Uncoilable and Failed Aneurysms (PUFS) trial

5. Commercial use in Europe (June 2008-present)

\section{EXPERIENCE FROM COMPASSIONATE USE}

Individual compassionate use cases have been performed in North America, South America (Buenos Aires) and Europe. Each of these cases has provided important proof of concept and served to advance our understanding of the potential uses and limitations of the device.

The first two patients treated in North America with PED both had circumferential vertebral artery aneurysms. Both patients were angiographically cured with Pipeline (three telescoping devices were used in each case) ${ }^{18}$ and both were neurologically normal, with 1year follow-up angiography demonstrating durable complete occlusion. These cases showed that the PED can provide a durable, curative, constructive endovascular treatment for lesions which present the greatest anatomical challenges to the existing commercially available endovascular devices. These cases also provided evidence that regional branch vessels covered by a PED construct (eg, anterior spinal artery, posterior inferior cerebellar arteries) can remain patent as the adjacent aneurysm progressively thromboses.

Recently, one of these original North American patients came to clinical attention just over 2 years after his treatment with vertebral occlusion at the level of the PED construct with an associated acute pontine infarct. He had spontaneously occluded the contralateral vertebral artery 2 years previously. This process began just over 1 week after local physicians stopped his aspirin therapy. This observation may indicate that these constructs retain some level of long term thrombogenicity and that at least single agent antiplatelet medication is required for life.

The third North American patient treated was a 13-year-old female with a giant circumferential symptomatic basilar artery aneurysm. ${ }^{19}$ This dramatic case example demonstrated that even the most daunting of lesions from a surgical or endovascular standpoint could be anatomically reconstructed with Pipeline. Moreover, the application of Pipeline in the basilar artery served to demonstrate that the regional perforating vessels proximal and distal to the aneurismal segment may remain patent when a PED construct is built judiciously to minimize the coverage over these eloquent segments. This patient remains neurologically normal, now 1 year out from Pipeline treatment. Follow-up CTA and MR have shown marked interval resorption of the associated thrombus mass with resolution of posterior fossa mass effect (fig 8). Follow-up angiography at 1 year demonstrates stable complete occlusion.

\section{PITA TRIAL}

The PITA triall ${ }^{22}$ was conducted at three European centers (Essen, Germany; Gratz, Austria; and Budapest, Hungary) and Buenos Aires, Argentina. In this study, 31 patients were treated. The lesions included in the PITA study were side wall aneurysms arising predominantly from the internal carotid artery (28 of 31) and were large in size (average $11.5 \mathrm{~mm}$ ) and wide necked (average $5.8 \mathrm{~mm}$ ). Two patients had stroke for an overall complication rate of $6 \%$. Of the 30 patients available for 6 month angiographic follow-up, 28 (93\%) were (angiographically) cured. No patients experienced delayed symptomatic in-stent stenosis and only one patient demonstrated angiographic in-stent stenosis which was greater than $25 \%$ but less than $50 \%$. This trial confirmed that for the population of side wall internal carotid aneurysms, Pipeline reproducibly provides a definitive, durable and curative endovascular treatment option that far exceeds the established track record for the predicate endosaccular techniques.

\section{BUENOS AIRES EXPERIENCE}

In Buenos Aires, the PED has been in use since March 2006 within the context of the PITA trial and under individual compassionate use provisions. During this time, Lylyk and colleagues ${ }^{11}$ have treated 63 aneurysms with the PED. The majority of aneurysms (70\%) were treated with a single device and in the vast majority of cases (94\%) the PED was used as a "standalone" device without the introduction of coils into the aneurysm. Six month angiographic follow-up demonstrated complete angiographic occlusion in $93 \%$ of patients. The lone aneurysm which remained patent after PED treatment at 12 months had been previously treated with stent supported coiling. Serial angiography never showed any deterioration in the angiographic result over time (ie, no "recurrence" or "recanalization" after treatment was observed).

These data provided further support for the hypothesis that the PED provides a durable curative endovascular treatment option for even the most challenging intracranial aneurysms. In addition, they indicate that a single PED typically provides enough coverage to achieve a curative reconstruction of most lesions, even as a "standalone" device without embolization coils. Finally, pre-existing endovascular constructs (eg, Neuroform (Boston Scientific), Enterprise (Cordis Neurovascular/ Johnson and Johnson), Wingspan (Boston Scientific)) may impair the wall apposition of the PED construct, reducing the ability of the device to induce a curative remodeling of the parent artery.

\section{INTERNATIONAL PIPELINE TRIALS (COMPLETE OCCLUSION OF COILABLE ANEURYSMS USING PIPELINE EMBOLIZATION DEVICE, COCOA AND PIPELINE EMBOLIZATION DEVICE FOR UNCOILABLE OR FAILED ANEURYSMS, PUFS)}

In October 2007, the first US patient was enrolled into the COCOA trial at the State University of New York at Stony Brook (SUNYSB) by Dr Henry Woo. At the time of the composition of this manuscript, both the COCOA and PUFS trials are actively underway at three institutions in the US (the Barrow Neurological Institute, New York University, SUNYSB) and a fourth in Budapest, Hungary (National Institute of Neurosurgery), with several additional sites expected to come online during the coming months.

COCOA is a randomized trial of endovascular coiling and PED reconstruction in "coilable" aneurysms of the petrous, cavernous and supraclinoid segments of the internal carotid artery. PUFS is 

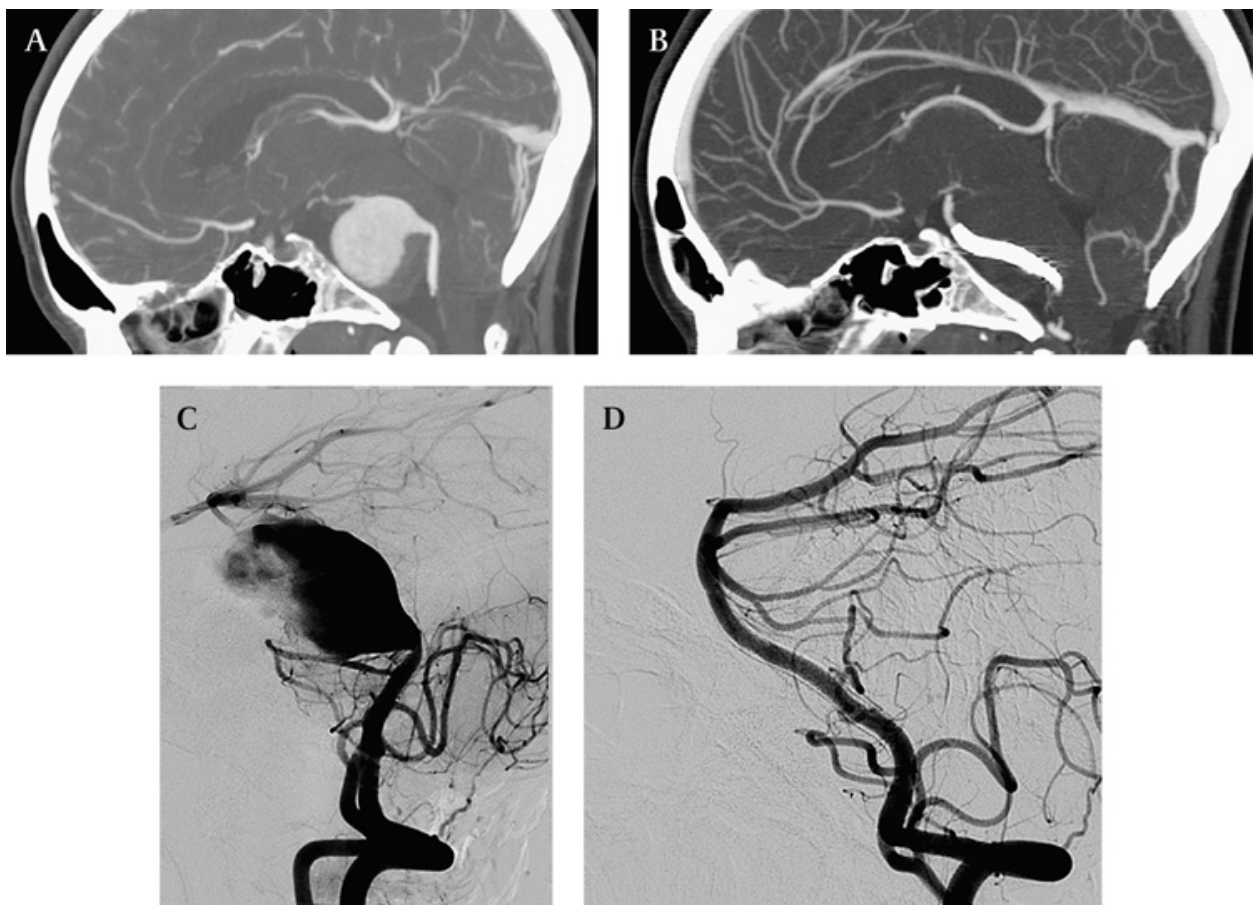

Figure 8 A 13-year-old female with a giant basilar trunk aneurysm presenting with headache. Pretreatment reconstructed sagittal image from CT angiography (CTA) source data show a $4 \mathrm{~cm}$ circumferential mid basilar aneurysm (A). This lesion was reconstructed with a Pipeline embolization device,and postoperative day 7 angiography demonstrated complete exclusion of the aneurysm. Follow-up CTA at 6 months (B) demonstrates near complete interval resorbtion of the associated thrombus mass with restoration of a near normal anatomical location of the basilar trunk. A tiny amount of residual intra-aneurysmal thrombus (measuring $7 \mathrm{~mm}$ in greatest thickness) remains along the anterior-inferior aspect of the lesion. A mass effect on the posterior fossa structures has also nearly completely resolved in the interim. The pretreatment angiogram (C) demonstrates the giant aneurysm and its mass effect on the basilar trunk with marked kinking of the vessel proximally, just distal to the vertebrobasilar junction. Follow-up angiography (D) at 1 year shows not only continued exclusion of the aneurysm but physiological restoration of a normal configuration of the basilar artery as the thrombus mass has been resorbed. The proximal kink now takes a smooth curvature and the distal basilar trunk is now normally aligned with the dorsal clivus.

a single arm trial of Pipeline for large $(>10 \mathrm{~mm})$ wide necked $(>4 \mathrm{~mm})$ aneurysms involving the same segments of the internal carotid artery.

Both trials incorporate a combined safety and efficacy endpoint, with efficacy defined as complete angiographic occlusion of the target aneurysm 180 days after treatment. The use of complete occlusion as the angiographic endpoint differentiates the PED trials from those for other endovascular aneurysm treatment devices which have been traditionally designed to measure a reduction in either recanalization or retreatment.

\section{COMMERCIAL USE IN EUROPE}

The PED received the CE mark in Europe on the basis of the PITA trial in June 2008. Since that time a limited number of patients have been treated and no results have yet been formally tracked or presented.

\section{THE FUTURE: EMERGENCE OF OTHER FLOW DIVERTING DEVICES}

Currently, there are a number of devices similar to the Pipeline which are in various stages of development or commercialization. Very little public information is available on these devices at this point. The Balt Silk stent (Balt Extrusion, Montmorency, France) has been approved for use in Europe since 2007. This is a hand woven braided construct similar to Pipeline. To date, no publications describing the results achieved with this device are available. The Surpass device (Surpass Medical, Tel Aviv, Israel) is a braided construct similar in structure to the PED.
Initial animal data evaluating the efficacy of the device to occlude experimental aneurysms in rabbits report similar outcomes to that of the PED. ${ }^{23} 24$ Certainly, the early clinical success of the Pipeline device will continue to stimulate interest in the development of additional similar devices which will be commercialized over the next decade.

\section{CONCLUSIONS: ANEURYSM TREATMENT WITH PIPELINE}

The current body of clinical experience with the Pipeline device has functioned to validate the conceptual basis for its development. As more case experience is accumulated and increasingly complex cases are undertaken, we will continue to advance our understanding of the Pipeline device and its role in the treatment of intracranial aneurysms.

The existing experience with the PED has indicated that:

1. PED achieves complete angiographic occlusion of unruptured side wall and fusiform-circumferential aneurysms. While the majority of aneurysms demonstrate some degree of residual contrast filling after "technically successful" coil occlusion which often persists (or worsens) during serial angiographic follow-up, the PED reliably achieves ( $>90 \%$ of the time) the endpoint of complete angiographic occlusion.

2. PED provides a durable treatment result. Aneurysm recurrence or "recanalization" represents a major limitation of aneurysm embolization with coils. ${ }^{25}$ In essence, some degree of aneurysm recurrence is expected with any endosaccular, endovascular approach to aneurysm treatment, 
particularly with larger, giant or wide necked aneurysms. ${ }^{10}$ This fundamental shortcoming represents the impetus behind the development of various surface modified coils, including coils coated with "bioactive" materials, expanding hydrogels and even radioactivity. ${ }^{26-28}$ In this regard, the Pipeline is again, fundamentally different, achieving a durable complete occlusion of these lesions. In fact, to date, there has never been a documented deterioration in the angiographic appearance of a saccular aneurysm treated with Pipeline that could be categorized as a recurrence or "recanalization" (PK Nelson, personal communication, 2009).

3. PED is optimal for some of the most challenging cerebral aneurysms. Large and giant wide necked and circumferential aneurysms represent some of the most difficult lesions to treat with standard open surgical and endovascular techniques. In fact, the aneurysm fundus size and neck width fundamentally define the technical difficulty, risk of complications and success rates involved with conventional endovascular (and to some degree surgical) aneurysm treatment. These factors become much less important when the endovascular strategy shifts from endosaccular coil occlusion to parent artery reconstruction. The existing data suggest that once the lesion is adequately bridged by a PED construct, complete occlusion will follow, regardless of the aneurysm fundus or neck size. Similarly, the durability of the occlusion achieved also does not seem to rely (at all) on the size of the lesion or width of the aneurysm neck.

4. PED is the only constructive endovascular technique which primarily reduces mass effect. Many large and giant aneurysms, particularly those arising from the internal carotid artery, present primarily with mass effect. The constructive endovascular treatment of these lesions with coils, liquid embolics and or commercially available stents does very little to reduce this mass effect. If these lesions recur multiple times, the addition of more and more coils can actually result in a gradual incremental exacerbation of the associated mass effect. The PED, by completely excluding these lesions from the circulation, allows the thrombus mass to be physiologically resorbed, resulting in the gradual reduction of the mass effect (and sometimes the edema) associated with these mass lesions (fig 8)

5. In-construct stenosis does not seem to be a significant issue (at least at this point). One potential issue with the implantation of endoluminal devices for the treatment of intracranial aneurysms is the potential risk of in-construct stenosis as the device becomes incorporated into the vessel and covered with neointima. This has rarely been a problem with the Neuroform (Boston Scientific) stent. ${ }^{29}$ When it occurs, it has typically been asymptomatic, often resolves spontaneously and very rarely requires re-intervention. With respect to the $\mathrm{PED}$, no cases of symptomatic in-construct stenosis have been documented and no asymptomatic in-construct stenosis has required endovascular or surgical re-intervention to date. One case of delayed symptomatic PED thrombosis has been observed but may have been related to the discontinuation of aspirin therapy or the sequela of an underlying vasculopathy.

6. Judicious coverage of regional perforators and larger branch vessels may be safe in some circumstances. In a small number of cases, PED constructs have been built across major branch arteries and/or regional perforators. Typically, operators have sought to limit the coverage of the regional perforators or branches to a single device at this point. While the existing data are too few to make a definitive statement about the safety of this practice, it does seem that in selected cases, this can be done safely.

7. Economics: The cumulative implantable costs associated with the coil embolization of large and giant aneurysms can be astronomical (fig 9). This is particularly true when one considers that these lesions tend to repeatedly recur, requiring multiple hospitalizations, retreatments and prolonged (essentially lifelong) imaging surveillance. At the same time, many of these large and giant aneurysms could be durably reconstructed with one or two PEDs. For this reason alone, the initial $\mathrm{PED}$ procedure could be performed at a fraction of the cost of endovascular coiling. The avoidance of multiple additional retreatments could further reduce the overall cost of aneurysm treatment, as well as the patient inconvenience and the risk of multiple interventions.

8. Radiation exposure. With the increasing attention being paid to the level of radiation exposure during diagnostic and interventional radiological procedures, ${ }^{30} 31$ it has become evident that our patients can be exposed to significant cumulative doses during treatment, subsequent follow-up and retreatments (if necessary). This is particularly true with large and giant aneurysms which require the placement of numerous embolization coils under high magnification fluoroscopic guidance during the original treatment and are also most likely to require subsequent angiographic follow-up and endovascular retreatment and endovascular retreatment. The number of PEDs required for aneurysm treatment is determined by the width of the aneurysm neck, rather than its overall size. The vast majority of aneurysms can be treated with fewer than three Pipeline devices. For these reasons, the PED reconstruction of most large and giant aneurysms can be accomplished in a fraction of the time which would be required to achieve angiographic occlusion with detachable coils (fig 10).

9. Pre-existing constructs may preclude or impair curative reconstruction with Pipeline. Pre-existing endovascular selfexpanding stents may complicate the delivery and deployment of the PED. These devices, even when seemingly optimally placed, may have individual tines projecting into the vessel lumen and may completely ovalize or kink in tortuous anatomy. ${ }^{32}$ These devices may make it more difficult to cross a lesion with one of the larger $(0.027$ inches internal diameter) microcatheters required for the delivery of the PED. They may also interfere with the proper deployment of the PED within the targeted segment. Finally, these pre-existing stents could prevent adequate apposition of the PED construct to the vessel wall, thereby creating an "endoleak" which can allow persistent filling of the aneurysm and prevent curative re-endothelialization of the treated vascular segment. For these reasons, operators may reconsider the elective stent supported treatment of some large, giant and wide necked aneurysms with conventional commercially available devices. These procedures may lead not only to incomplete or non-durable treatments with considerable associated periprocedural risk, but they may also preclude a curative reconstruction of the lesion in the future with Pipeline.

While we have some insight into the potential role of the Pipeline device in the treatment of cerebral aneurysms, several important limitations in our understanding of the device persist.

1. Use in subarachnoid hemorrhage. Many of the aneurysms which come to clinical attention, and those which 

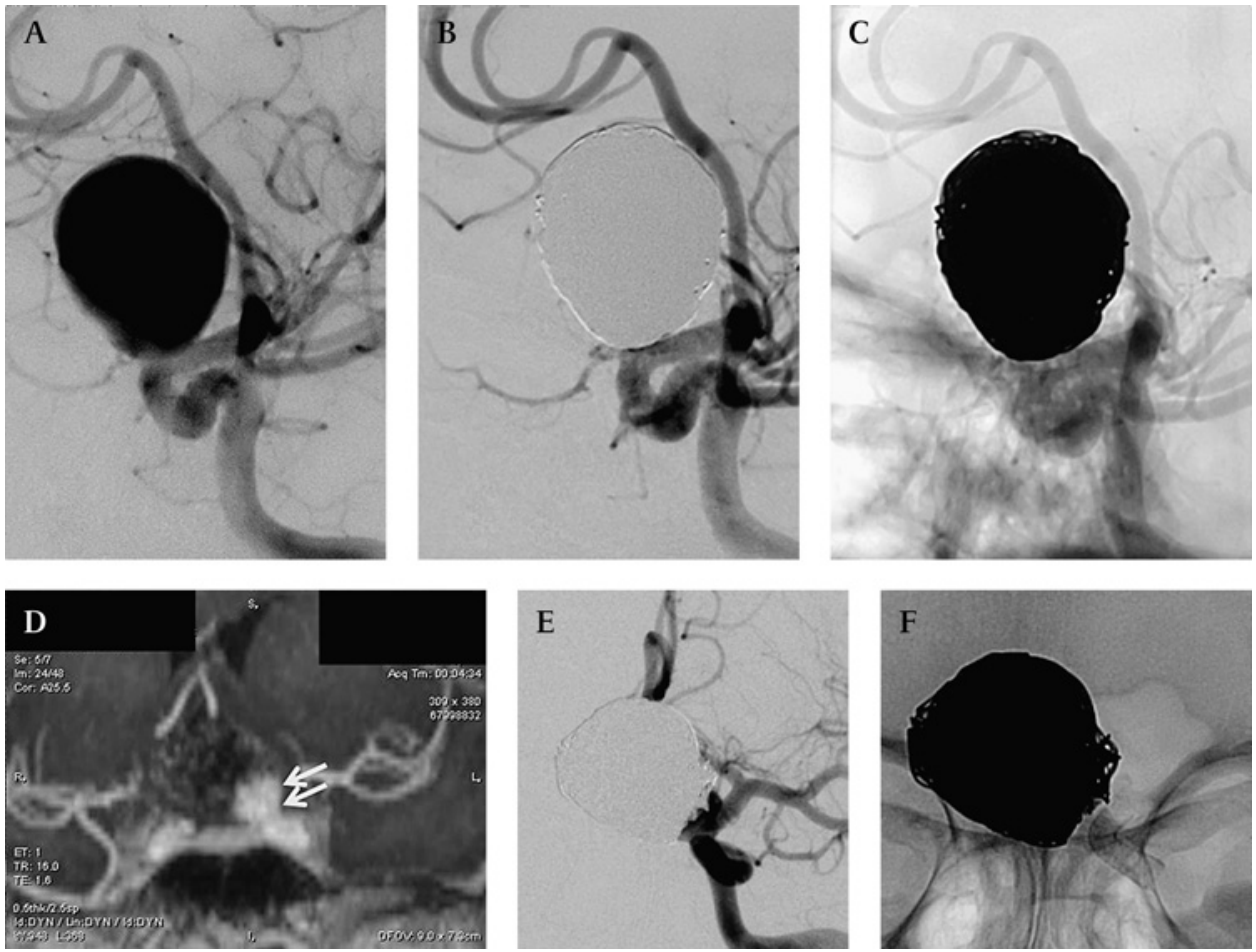

Figure 9 A 46-year-old female with a giant left internal carotid artery carotid-ophthalmic aneurysm symptomatic with headaches and left eye vision impairment. She originally presented before the initiation of the US Pipeline embolization device (PED) trials and was treated with balloon assisted coil embolization. Subtracted pretreatment $(A)$ and immediate post-treatment subtracted $(B)$ and native $(C)$ images in the working angles for coil embolization show the giant aneurysm to be densely packed with coils and completely occluded at the conclusion of the procedure. The total cost of coils for the case was over 80000 US\$. Her symptoms improved over the next several weeks. However, she presented 7 months later with recurrent headaches and visual decline. A coronal reconstruction from a contrast enhanced short echo time MR angiography (D) shows a large recurrence (arrows) at the aneurysm neck with coil compaction into the aneurysm fundus. This recurrence was confirmed angiographically (E) and treated with PED within the context of the PUFS trial. A native image $(F)$ following treatment demonstrates a PED construct in place across the aneurysm neck. It is possible that such lesions will be easily amenable to durable curative reconstruction with one or two PEDs. This represents a tremendous potential saving to the health care system in terms of reducing the overall cost of implantables as well as the need for multiple retreatments.

unambiguously require treatment, are acutely ruptured. Because of the requirement for dual antiplatelet medications to maintain the patency of the Pipeline device, the application of this technology in patients with acute subarachnoid hemorrhage remains unknown. Experience with the commercially available self-expanding stents would suggest that the role of the PED in subarachnoid hemorrhage is likely to be very limited. ${ }^{33}$

2. Use in bifurcation anatomy. The safety and efficacy of using the PED to treat bifurcation aneurysms remain unknown at this time. This will surely be defined as experience with the device continues to expand.
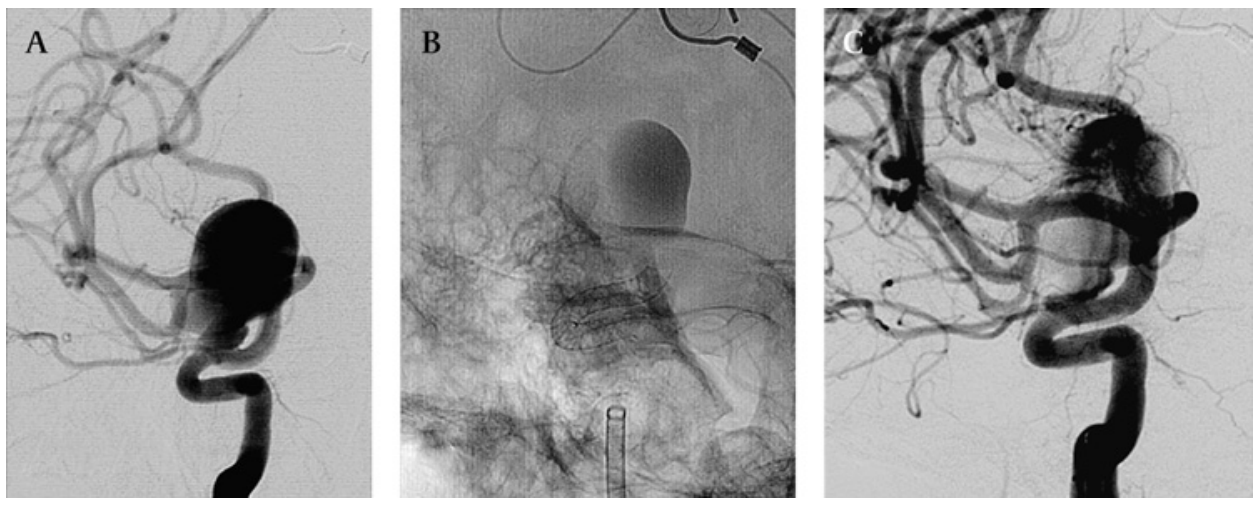

Figure 10 A 71-year-old female with multiple large intracranial aneurysms. Subtracted (A) right internal carotid artery (ICA) angiogram in the working angle for reconstruction with a Pipeline embolization device (PED) demonstrates an irregular, $18 \mathrm{~mm}$ carotid-ophthalmic segment aneurysm. A native (B) image after reconstruction shows two PEDs in place within the supraclinoid ICA and contrast stasis within the dependent aspect of the aneurysm. The subtracted image (C) shows very effective flow diversion with minimal residual filling of the aneurysm. Coil embolization of this aneurysm likely would have required either a balloon assisted or stent assisted technique. At least 15-20 coils would have been required to achieve an adequate occlusion of the aneurysm. Each of these coils would have required continuous fluoroscopic visualization for placement and detachment. Typically a case of this complexity with an aneurysm of this size would require more than $1 \mathrm{~h}$ of fluoroscopic time. The reconstruction of this lesion with Pipeline required a total of only $16 \mathrm{~min}$ of fluoroscopic time. 
3. Long term effects (patency, thrombogenicity, durability), The Pipeline has been used in humans for only 2 years, with the vast majority of cases having been performed over the past 6-9 months. As such, the long term effects of the device within the human cerebrovasculature remain largely unknown. The appropriate duration of dual (and then single) antiplatelet therapy required to maintain the patency of the device and any covered side branches likewise remains uncertain.

Over the next several years, the Pipeline, and possibly other similar flow diverting devices, will radically change the manner in which cerebral aneurysms are treated. It is appears that we will be able to achieve more definitive and durable results than ever before using endovascular techniques. Furthermore, these devices will allow us to treat lesions which cannot be adequately or safely approached by the current armamentarium of endovascular devices.

Competing interests: PN is a consultant and stockholder in Chestnut Medical.

\section{REFERENCES}

1. Molyneux A, Kerr R, Stratton I, et al. International Subarachnoid Aneurysm Trial (ISAT) of neurosurgical clipping versus endovascular coiling in 2143 patients with ruptured intracranial aneurysms: a randomised trial. Lancet 2002;360:1267-74.

2. Molyneux AJ, Kerr RS, Yu LM, et al. International Subarachnoid Aneurysm Trial (ISAT) of neurosurgical clipping versus endovascular coiling in 2143 patients with ruptured intracranial aneurysms: a randomised comparison of effects on survival, dependency, seizures, rebleeding, subgroups, and aneurysm occlusion. Lancet 2005;366:809-17.

3. McDougall CG. Barrow Ruptured Aneurysm Trial: one year results. American Association of Neurological Surgeons Annual Meeting, 20-22 Feb 2008. Chicago, Illinois, 2008.

4. Johnston SC, Zhao S, Dudley RA, et al. Treatment of unruptured cerebral aneurysms in California. Stroke 2001;32:597-605.

5. Albuquerque FC, Fiorella D, Han P, et al. A reappraisal of angioplasty and stenting for the treatment of vertebral origin stenosis. Neurosurgery 2003;53:607-14.

6. Fiorella D, Albuquerque FC, Deshmukh VR, et al. Usefulness of the Neuroform stent for the treatment of cerebral aneurysms: results at initial (3-6-mo) follow-up. Neurosurgery 2005;56:1191-201.

7. Fiorella D, Albuquerque FC, Masaryk TJ, et al. Balloon-in-stent technique for the constructive endovascular treatment of "ultra-wide necked" circumferential aneurysms. Neurosurgery 2005;57:1218-27.

8. Raymond J, Guilbert F, Weill A, et al. Long-term angiographic recurrences after selective endovascular treatment of aneurysms with detachable coils. Stroke 2003; 34:1398-403.

9. Murayama Y, Nien YL, Duckwiler G, et al. Guglielmi detachable coil embolization of cerebral aneurysms: 11 years' experience. J Neurosurg 2003;98:959-66.

10. van Rooij WJ, Sluzewski M. Endovascular treatment of large and giant aneurysms AJNR Am J Neuroradiol 2009;30:12-18.

11. Lylyk P, Ferrario A, Pasbon B, et al. Buenos Aires experience with the Neuroform self-expanding stent for the treatment of intracranial aneurysms. J Neurosurg 2005; 102:235-41.

12. Lylyk $\mathbf{P}$, Cohen JE, Ceratto $\mathrm{R}$, et al. Endovascular reconstruction of intracranial arteries by stent placement and combined techniques. J Neurosurg 2002;97:1306-13.
13. Sluzewski M van Rooij WJ Slob MJ, et al. Relation between aneurysm volume, packing, and compaction in 145 cerebral aneurysms treated with coils. Radiology 2004;231:653-8.

14. Wakhloo AK, Gounis MJ, Sandhu JS, et al. Complex-shaped platinum coils for brain aneurysms: higher packing density, improved biomechanical stability, and midterm angiographic outcome. AJNR Am J Neuroradiol 2007:28:1395-400.

15. Fiorella D, Woo H, Albuquerque F, et al. Definitive reconstruction of circumferential fusiform intracranial aneurysms with the Pipeline embolization device. Neurosurgery 2008;62:1115-20.

16. Kallmes DF, Ding YH, Dai D, et al. A new endoluminal, flow-disrupting device for treatment of saccular aneurysms. Stroke 2007:38:2346-52.

17. Fiorella D, Kelly ME, Turner RD, et al. Endovascular treatment of cerebral aneurysms. . . Endovascular Today 2008 June;53-65.

18. Fiorella D, Woo HH, Albuquerque FC, et al. Definitive reconstruction of circumferential, fusiform intracranial aneurysms with the Pipeline embolization device. Neurosurgery 2008:62:1115-20.

19. Fiorella D, Kelly ME, Albuquerque FC, et al. Curative reconstruction of a giant midbasilar trunk aneurysm with the Pipeline embolization device. Neurosurgery 2009;64:212-17.

20. Lopes DK, Ringer AJ, Boulos AS, et al. Fate of branch arteries after intracranial stenting. Neurosurgery 2003;52:1275-8.

21. Wakhloo AK, Tio FO, Lieber BB, et al. Self-expanding nitinol stents in canine vertebral arteries: hemodynamics and tissue response. AJNR Am J Neuroradiol 1995;16:1043-51.

22. Nelson PK. Pipeline for the Intracranial Treatment of Aneurysms (PITA) trial. International Stroke Conference (ISC). New Orleans (LA), USA, 20-22 Feb 2008.

23. Sadasivan C, Cesar L, Seong J, et al. Treatment of rabbit elastase-induced aneurysm models by flow diverters: development of quantifiable indices of device performance using digital subtraction angiography. IEEE Trans Med Imaging, 2009. (Epub ahead of print 19 Jan 2009) doi:10.1109/TMI.2008. 2012162.

24. Sadasivan C, Cesar L, Seong J, et al. An original flow diversion device for the treatment of intracranial aneurysms: evaluation in the rabbit elastase-induced model. Stroke 2009; 40:952-8.

25. White PM, Lewis SC, Nahser H, et al. HydroCoil Endovascular Aneurysm Occlusion and Packing Study (HELPS trial): procedural safety and operator-assessed efficacy results. AJNR Am J Neuroradiol 2008;29:217-23.

26. Raymond J, Mounayer C, Salazkin I, et al. Safety and effectiveness of radioactive coil embolization of aneurysms: effects of radiation on recanalization, clot organization, neointima formation, and surrounding nerves in experimental models. Stroke 2006;37:2147-52

27. Fiorella D, Albuquerque FC, McDougall CG. Durability of aneurysm embolization with matrix detachable coils. Neurosurgery 2006:58:51-9.

28. Cloft HJ. HydroCoil for Endovascular Aneurysm Occlusion (HEAL) study: 3-6 month angiographic follow-up results. AJNR Am J Neuroradiol 2007;28: $152-4$

29. Fiorella D, Albuquerque FC, Woo $\mathrm{H}$, et al. Neuroform in-stent stenosis: incidence, natural history, and treatment strategies. Neurosurgery 2006;59:34-42.

30. Rooij WJ, Keeren GJ, Peluso JP, et al. Clinical and angiographic results of coiling of 196 very small $(\leq 3 \mathrm{~mm})$ intracranial aneurysms. AJNR Am J Neuroradiol 2009;30:835-9.

31. Weerakkody RA, Walsh SR, Cousins C, et al. Radiation exposure during endovascular aneurysm repair. Br J Surg 2008;95:699-702.

32. Ebrahimi N, Claus B, Lee $\mathrm{CY}$, et al. Stent conformity in curved vascular models with simulated aneurysm necks using flat-panel CT: an in vitro study. AJNR Am J Neuroradiol 2007:28:823-9.

33. Tumialan LM, Zhang YJ, Cawley CM, et al. Intracranial hemorrhage associated with stent-assisted coil embolization of cerebral aneurysms: a cautionary report. J Neurosurg 2008:108:1122-9. 